\title{
APPLICATION DE LA MÉCANIQUE PLASTIQUE DES RUPTURES A LA PRÉVISION DE LA TAILLE DE DÉFAUTS CRITIQUES
}

\author{
G. PLUVINAGE Université de Metz \\ Y. LECOINTE Ecole Nationale Technique des Mines de Douai \\ et F. MONTARIOL Université de Lille
}

\begin{abstract}
Résumé. - La mécanique plastique des ruptures permet une sélection des aciers à basse et moyenne résistance en vue de se prémunir du risque de rupture fragile. Elle utilise pour cela le critère d'écartement critique de fissuration (C. O. D.). L'écartement critique de fissuration et la déformation globale autour d'un défaut sont reliés par une relation linéaire dans le domaine de validité du C. O. D. Nous avons vérifié ceci sur de larges plaques d'acier en $8 \mathrm{~mm}$ d'épaisseur, contenant un défaut artificiel et soumises à une contrainte de traction. On peut déterminer à partir de cette relation et de la ténacité de l'acier, la taille de défaut admissible pour un niveau de contrainte donné.

Abstract. - Plastic fracture mechanics can allowed low and middle strength steel's selection in order of fracture safe design. It takes in consideration, critical crack tip opening displacement (C. O. D.). Critical crack tip opening displacement and overall strain throught a defect give a linear relation ship in C. O. D.'s validity zone. We should give an experimental verification of this paint with large steel's sheets with artificial defect and stressed in tension. Man can made calculation of critical defect size with aid of this relation and steel's toughness of a given stress level.
\end{abstract}

La sélection d'un matériau en vue de la réalisation d'une construction soumise à des sollicitations complexes (par exemple un réservoir sous pression) impose aux bureaux d'études de se conformer à l'article 4 de l'Arrêté Ministériel du 23 juillet 1943 :

" L'ouvrage ne doit pas être fragile dans les conditions de service, ni dans les conditions de l'épreuve hydraulique. »

Le déclenchement d'une rupture fragile nécessite l'existence des 3 conditions suivantes:

- la température de service est inférieure à une "température de transition »; nous définirons cette notion de température de transition ultérieurement;

- le niveau de contrainte est suffisamment élevé dans la région considérée de l'ouvrage;

- on se trouve en présence d'un défaut dont l'acuité permet d'initier une rupture fragile.

Ces trois conditions présentes simultanément risquent de produire une rupture fragile ; on est à l'abri de ce risque si l'une d'entre elles n'est pas remplie, c'est-à-dire si la température est assez élevée, s'il n'existe aucune contrainte ou si on constate l'absence d'entaille.

On remarque l'existence d'interactions entre ces conditions, ainsi des défauts plus aigus, des contraintes en service plus élevées, augmentent la température à laquelle ce risque de rupture est susceptible de se produire.
L'ensemble de ces 3 paramètres: Température, contrainte et taille de défaut peut définir la ténacité d'un matériau. En fixant deux de ces paramètres, on se donne un critère de ductilité car le troisième est imposé par les caractéristiques du matériau et dans une construction la rupture se produit lorsque ces trois grandeurs atteignent les valeurs critiques.

Cette notion est restée et reste encore purement conceptuelle mais l'introduction récente de la mécanique des ruptures tend à lui donner une formulation.

Le taux de travail maximal est fixé par le législateur :

- au tiers de la résistance à la traction du métal à la température ordinaire ;

- au cinq huitièmes de la limite d'élasticité à $0,2 \%$ de ce métal à la température maximale en service.

Ce niveau de contrainte défini, le bureau d'études doit alors se soucier de savoir si la température de service n'est pas en dessous d'une température limite marquant le passage d'une rupture par décohésion (rupture fragile) à une rupture se produisant après une déformation plastique importante même en présence d'entaille (rupture ductile), c'est-à-dire que la température de service ne soit pas en dessous d'une certaine température de transition.

La définition expérimentale d'une température de 
transition est assez délicate, notamment parce que celle du matériau peut être différente de celle de la construction ; on la situe plus aisément pour les aciers à basse et moyenne résistance pour lesquels la température a une influence importante sur la ténacité, les aciers à haute résistance ont une variation nettement moins marquée.

De très nombreuses méthodes d'essai existent pour définir cette température de transition, elles diffèrent par la forme et les dimensions de l'éprouvette, le mode de sollicitation ; citons, par exemple, les essais de résilience, de traction par choc, essais Robertson, S. O. D., essai Kinsel, Van der Veen, Pellini, etc... Cependant l'essai de résilience Charpy $\mathrm{V}$ reste la méthode la plus couramment employée parce que la plus simple et la moins onéreuse. On considère généralement la température de transition comme celle où l'énergie de rupture atteint :

- 3,5 $\mathrm{daJ} / \mathrm{cm}^{2}$ pour les aciers non alliés A37, A42, A48,

- $5 \mathrm{daJ} / \mathrm{cm}^{2}$ pour les aciers au carbone et au carbone manganèse à 52/60 hbar de résistance à la rupture.

Cependant l'essai de résilience ne met pas en évidence l'influence de l'épaisseur, ainsi d'après Wells [1] l'énergie de rupture devrait croître :

- proportionnellement à l'épaisseur de la structure envisagée ;

- proportionnellement au carré de la limite d'élasticité.

Dans un troisième temps et surtout au moment de la mise en œuvre il est important de se soucier de l'existence de défauts ou d'effets d'entailles (dus aux variations à angle vif de la forme et de l'épaisseur de la paroi, à l'existence de raidisseurs, d'attaches, de supports).

Les défauts peuvent aussi provenir des soudures ou des variations brutales des propriétés mécaniques.

Une construction, et surtout une construction soudée, est difficilement réalisable sans défaut, aussi les codes de constructions tolèrent-ils de très petits défauts. $\mathrm{Du}$ fait de leur existence inéluctable, 3 questions se posent à leur propos :

1) Quelle est la dimension critique du défaut (c'està-dire quelle est sa taille requise pour provoquer la rupture au niveau de contrainte en service ?).

2) Quelle est la taille maximale du défaut qui existe dans la construction avant sa mise en service ?

3) Comment ce défaut initial va-t-il croître jusqu'à sa taille critique et causer la rupture durant la vie en service de cette construction?

Les réponses à ces questions dépendent de la résistance à la rupture fragile du matériau, mais aussi des facteurs de sécurité adoptés, des essais sous pression, et aussi des examens non destructifs.

Des récentes études [2] ont montré l'utilisation de la mécanique linéaire des ruptures dans la prédiction de la taille de défaut critique et l'estimation de la durée de vie de ces constructions.

Cette mécanique linéaire des ruptures définit un facteur d'intensité de contrainte $K_{1}$ à la pointe d'un défaut en fonction de la contrainte appliquée $\sigma$ et de la dimension de ce défaut à :

$$
K_{1}=\alpha \sigma \sqrt{\pi a}
$$

$\alpha$ étant un coefficient qui dépend de la géométrie de l'éprouvette et de la longueur de fissure.

Le critère de rupture est alors un critère de contrainte et on pense que la rupture se produit lorsque le facteur d'intensité de contrainte atteint une valeur critique $K_{1 \mathrm{c}}$. Cette valeur $K_{1 \mathrm{c}}$ est une caractéristique de la résistance d'un matériau à la propagation brutale d'une fissure par ouverture des lèvres de celle-ci dans un champ de déformation plane (et seulement lorsque cette condition est remplie).

Il existe une relation entre longueur de fissure critique $a_{\mathrm{c}}$ et le facteur d'intensité critique de contrainte $K_{1 \mathrm{c}}$, relation donnée par la formule :

$$
a_{\mathrm{c}}=K_{1 \mathrm{c}}^{2} \frac{\left[\varphi^{2}-0,212\left(\sigma / \sigma_{y}\right)^{2}\right]}{1,21 \pi \sigma^{2}}
$$

avec $\varphi$ intégrale elliptique :

$$
\int_{0}^{\pi / 2}\left(1-\frac{c^{2}-a^{2}}{c^{2}} \sin ^{2} \theta\right) d \theta .
$$

La fissure étant assimilée à une ellipse très aplatie de grand axe $2 c$ et de petit axe $2 a$.

Cette théorie de la mécanique des ruptures a donné quelques bons résultats notamment sur des aciers à haute limite d'élasticité et des vérifications de la prévision de la taille de défaut critique ont été faites notamment par Tifany et Masters [3].

Les conditions d'applications de la mécanique linéaire des ruptures doivent satisfaire aux exigences d'un régime de déformation plane et d'une zone plastifiée en fond de fissure petite, il est difficile de réaliser ces conditions avec des aciers à moyenne résistance sans utiliser de très grosses éprouvettes ; on doit alors faire appel à la théorie de l'écartement critique de fissuration.

1. Théorie de l'écartement critique de fissuration. La théorie de l'écartement critique de fissuration (en anglais : Crack-opening displacement, abréviation C. O. D.) fut d'abord formulée par Wells [4] : l'écartement critique des lèvres d'une fissure est considéré comme un critère de la résistance à l'amorçage des déchirures et fut développé en particulier par Burdekin [5].

Cette théorie de l'écartement critique de fissuration est surtout applicable dans le cas des aciers où la zone plastifiée en fond de fissure devient importante et rend possible l'écartement des lèvres de la fissure sans accroissement de sa longueur. 
En cela la théorie de l'écartement critique de fissuration prolonge la mécanique linéaire des ruptures qui ne tolère qu'une faible déformation plastique et reste d'un emploi limité aux aciers à haute limite d'élasticité (Fig. 1).

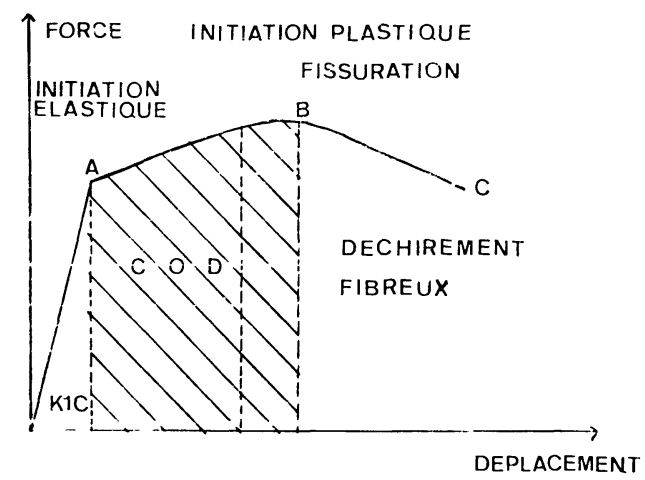

FIG. 1. - Représentation schématique des domaines de validité des essais de ténacité pour les ruptures entre $\mathbf{A}$ et $\mathbf{B}$ on utilise la théorie de l'écartement critique de fissuration.

A l'aide du modèle à " couche plastique " proposé par Dugdale et Barenblatt [6-7], on peut relier l'écartement critique de fissuration $\delta_{c}$ et l'énergie de fissuration par unité de surface $G_{\mathrm{c}}$.

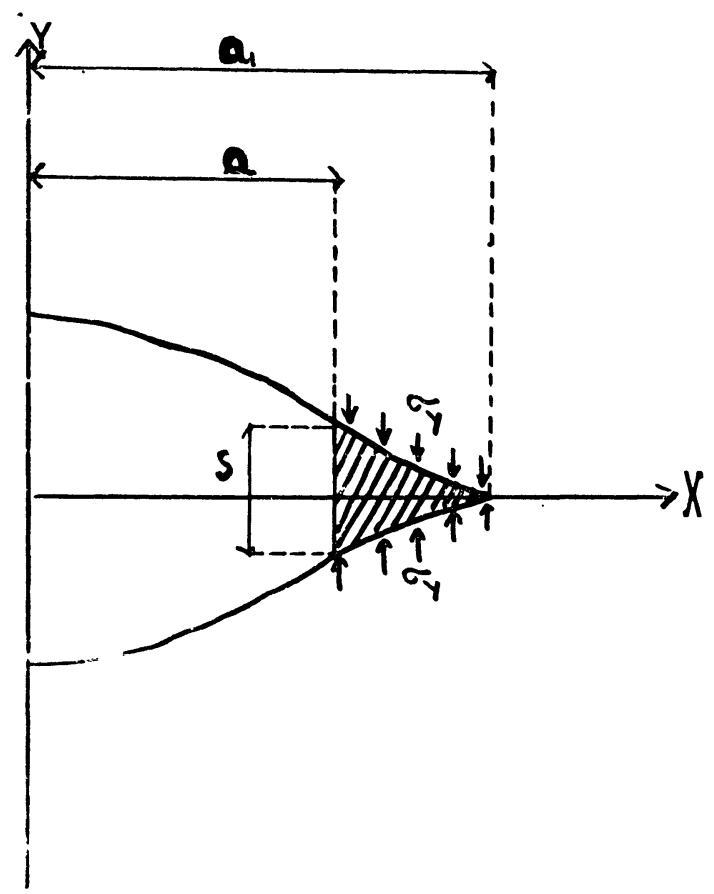

Fig. 2. - Modèle de Dugdale-Barenblatt.

Ce modèle représenté dans la figure 2 est employé dans l'analyse théorique qui considère une fissure de longueur $2 a$ dans une plaque infinie. Sous l'effet d'une contrainte $\sigma$ appliquée dans une direction normale à la fissure, des zones plastifiées se développent et s'étendent jusqu'en $x= \pm a_{1}$.
On considère alors, pour l'analyse, une fissure de longueur $a_{1}$ entourée par un champ de contrainte élastique $\sigma$ et aussi des contraintes de compression dans la direction $y$ d'amplitude $\sigma_{y}\left(\sigma_{y}\right.$ limite d'élasticité du matériau) réparties sur la longueur $\left(a_{1}-a\right)$.

Les calculs de Burdekin et Stone [5] montrent que l'écartement de fissure $\delta$ (au fond de la fissure réelle) est donné par le déplacement en $x \pm a$ est égal à :

$$
\delta=\frac{8 \sigma_{y} a}{\pi E} \log _{\mathrm{e}} \sec \left[\frac{\pi \sigma}{2 \sigma_{y}}\right] .
$$

De même la déformation globale sur une longueur de jauge $2 y$ est égale à : (la longueur de jauge est la longueur de la base de mesure de la déformation globale)

$$
\begin{aligned}
e_{\mathrm{g}}= & \frac{2 E}{\sigma_{y}}\left\{2 n \operatorname { c o t h } ^ { - 1 } \left[\frac{1}{n} \sqrt{\frac{k^{2}+n^{2}}{1-k^{2}}}+\right.\right. \\
& \left.\left.+1-v \cot ^{-1} \sqrt{\frac{k^{2}+n^{2}}{1-K^{2}}}-v \cos ^{-1} k\right]\right\}
\end{aligned}
$$

avec

$$
n=\frac{a}{y} \quad \text { et } \quad k=\frac{a}{a_{1}} \cos \left[\frac{\pi \sigma}{2 \sigma_{y}}\right]
$$

On peut d'autre part relier l'écartement critique de fissuration à l'énergie de fissuration par unité de surface $G_{\mathrm{c}}$ qui est égale en mécanique linéaire des ruptures, lorsque la zone plastique est petite, à :

$$
G_{\mathrm{c}}=\frac{\pi \sigma^{2} a}{E}
$$

En effet l'éq. (3) peut se mettre sous la forme d'un développement limité :

$$
\begin{aligned}
\delta=\frac{8 \sigma_{y} a}{E}\left\{\frac{1}{2}\left[\frac{\pi \sigma}{2 \sigma_{y}}\right]^{2}+\frac{1}{12}\left[\frac{\pi \sigma}{2 \sigma_{y}}\right]^{4}\right. & + \\
& \left.+\frac{1}{45}\left[\frac{\pi \sigma}{2 \sigma_{y}}\right]^{6}+\cdots\right\}
\end{aligned}
$$

en ne considérant que le premier terme

$$
\delta=\frac{\pi \sigma^{2} a}{E \sigma_{y}} .
$$

En comparant les éq. (5) et (6) on obtient la relation :

$$
G_{\mathrm{c}}=\sigma_{y} \delta \text { quand } \sigma / \sigma_{y}<1 .
$$

Notons qu'il est commode d'utiliser un écartement critique de fissuration non dimensionnel uniquement fonction du rapport $\sigma / \sigma_{y}$

$$
\Phi=\frac{\delta}{2 \pi \varepsilon_{y} a}=\frac{4}{\pi^{2}} \log _{\mathrm{e}} \operatorname{Sec}\left[\frac{\pi \sigma}{2 \sigma_{y}}\right]^{2} .
$$

Avec $\varepsilon_{y}$ la déformation à la limite élastique $\varepsilon_{y}=\sigma_{y} / E$. 
2. Etude expérimentale de la relation entre l'écartement critique de fissuration et la déformation globale autour d'un défaut. - Les mesures d'écartement critique de fissuration se font habituellement en laboratoire sur de petites éprouvettes [8]. La technique de mesure la plus classique utilise une éprouvette parallélépipédique à base carrée entaillée à mi-longueur d'une entaille profonde du tiers de la section, entaille terminée par une fissure de fatigue. L'éprouvette montée sur rouleaux est sollicitée en flexion trois points. L'écartement des lèvres de l'entaille est mesuré à l'aide d'un extensomètre à lames. Par étalonnage ou par calcul, on déduit des indications de cet extensomètre la valeur de l'écartement correspondant à fond de fissure au moment de la rupture ; cet écartement est alors identifié à sa valeur critique (Fig. 3).

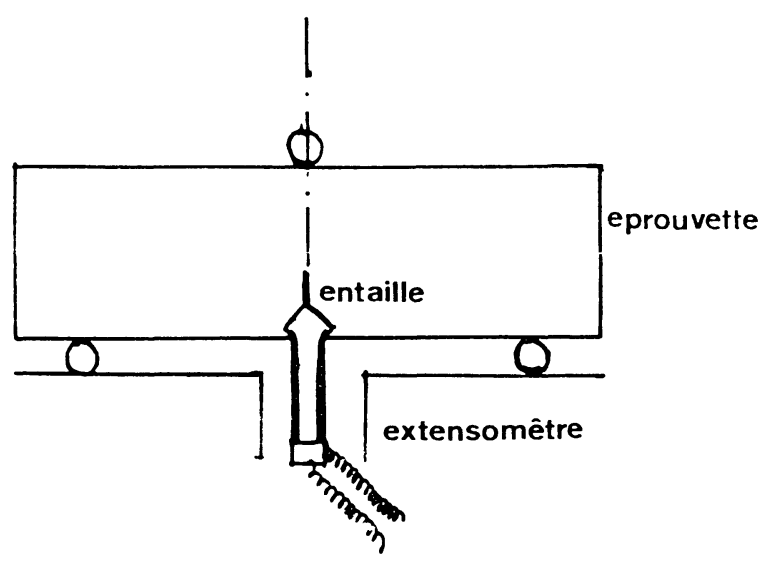

Fig. 3. - Schéma de montage pour la mesure de l'écartement de fissure.

Cet essai destructif ne se prête pas toujours au contrôle en service des structures; la déformation globale autour d'un défaut d'une telle structure est généralement plus aisément mesurable, certains auteurs [9] pensant même l'utiliser pour apprécier la ténacité de constructions possédant de gros défauts.

Déformation globale et écartement de fissure non dimensionnel sont liés entre eux par l'intermédiaire du rapport de la contrainte appliquée à la contrainte, à la limite d'élasticité et l'utilisation de telles courbes $\Phi=\rho\left(e_{\mathrm{g}}\right)$ est intéressante du point de vue de la sécurité dans la conception des constructions. Cependant les considérations de Burdekin et Stone omettent l'influence de l'écrouissage, de la triaxialité des contraintes et des dimensions finies de l'éprouvette ; aussi avonsnous pensé faire la comparaison entre cette analyse théorique et une simulation expérimentale sur plaques de dimensions très supérieures aux éprouvettes habituellement utilisées en laboratoire.

2.1 MATÉRIAU UTILISÉ. - Nous avons utilisé un acier de construction non allié que nous avons reçu sous forme de tôles laminées d'épaisseur $8 \mathrm{~mm}$. Cet acier était du type A 42.
Le tableau suivant nous indique sa composition chimique :

$\begin{array}{ccccc}\mathrm{C} & \mathrm{Mn} & \mathrm{Si} & \mathrm{S} & \mathrm{P} \\ \overline{0,16} & - & - & - & - \\ 0,46 & & 0,28 & 0,022 & 0,02\end{array}$

Nous avons relevé les propriétés mécaniques suivantes :

- contrainte à la limite d'élasticité 30,2 hbar,

- contrainte de rupture 42,5 hbar,

- énergie de rupture sur éprouvette en long prise dans l'épaisseur du produit $(8 \times 10 \times 55 \mathrm{~mm})$ avec entaille en V $2 \mathrm{~mm}$ à $20^{\circ} \mathrm{C}: 4,65 \mathrm{daJ}$.

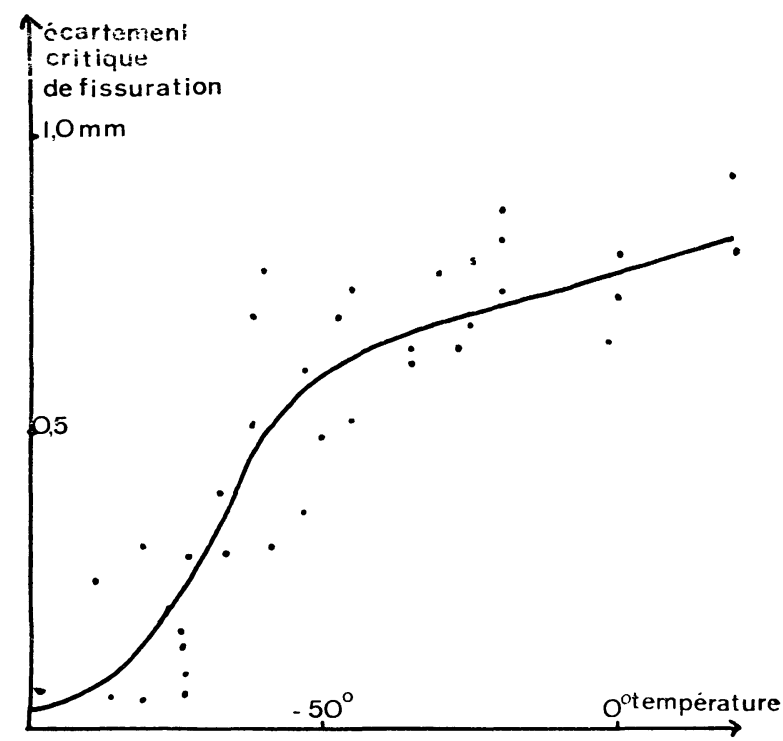

Fig. 4. - Courbe d'écartement critique de fissuration en fonction de la température d'essai (acier type A 42).

La courbe de la figure 4 indique les variations de l'écartement critique de fissuration en fonction de la température. Cet écartement critique de la fissuration a été mesuré à l'aide de petites éprouvettes prélevées dans la pleine épaisseur de la tôle.

2.2 GÉOMÉTRIE DES ÉPROUVETTES. - Nous avons utilisé pour nos essais 5 séries de plaques aux dimensions suivantes : $300 \times 300 \mathrm{~mm} ; 400 \times 400 \mathrm{~mm}$; $500 \times 500 \mathrm{~mm} ; 600 \times 600 \mathrm{~mm}$ et $800 \times 800 \mathrm{~mm}$.

Dans le centre de ces plaques et perpendiculairement à la direction de sollicitation, nous avons pratiqué des défauts artificiels dont la géométrie est donnée par la figure 5. Les plaques furent sollicitées en traction à l'aide d'une machine de capacité de $60 \mathrm{t}$.

Pour mesurer l'écartement des lèvres du défaut, nous avons utilisé un capteur à lames flexibles, maintenu sur la plaque à l'aide de deux petites cales vissées à une distance de $20 \mathrm{~mm}$ des lèvres du défaut (photo $\mathrm{n}^{\mathrm{o}}$ 1).

On enregistre la déformation du capteur et un étalonnage à l'aide d'un comparateur au 1/100 permet de déduire l'écartement des bords de ce capteur (Fig. 6). 


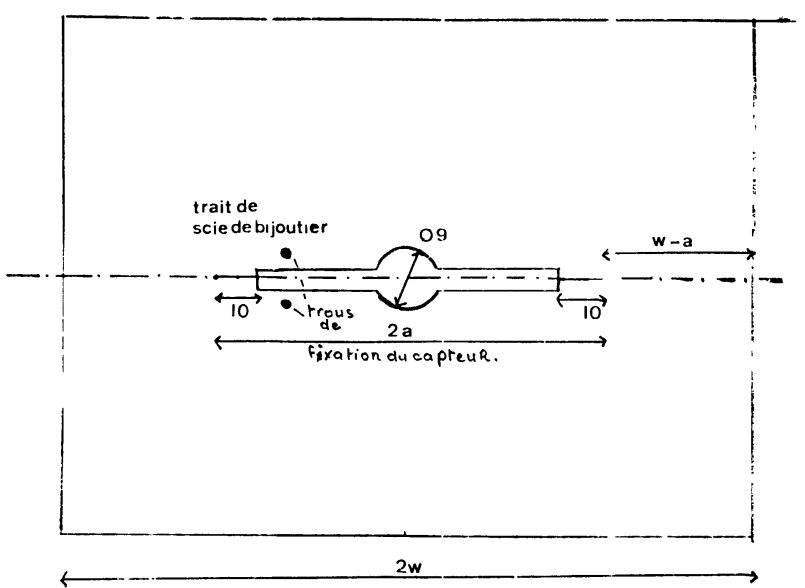

Fig. 5. - Dessin des éprouvettes.

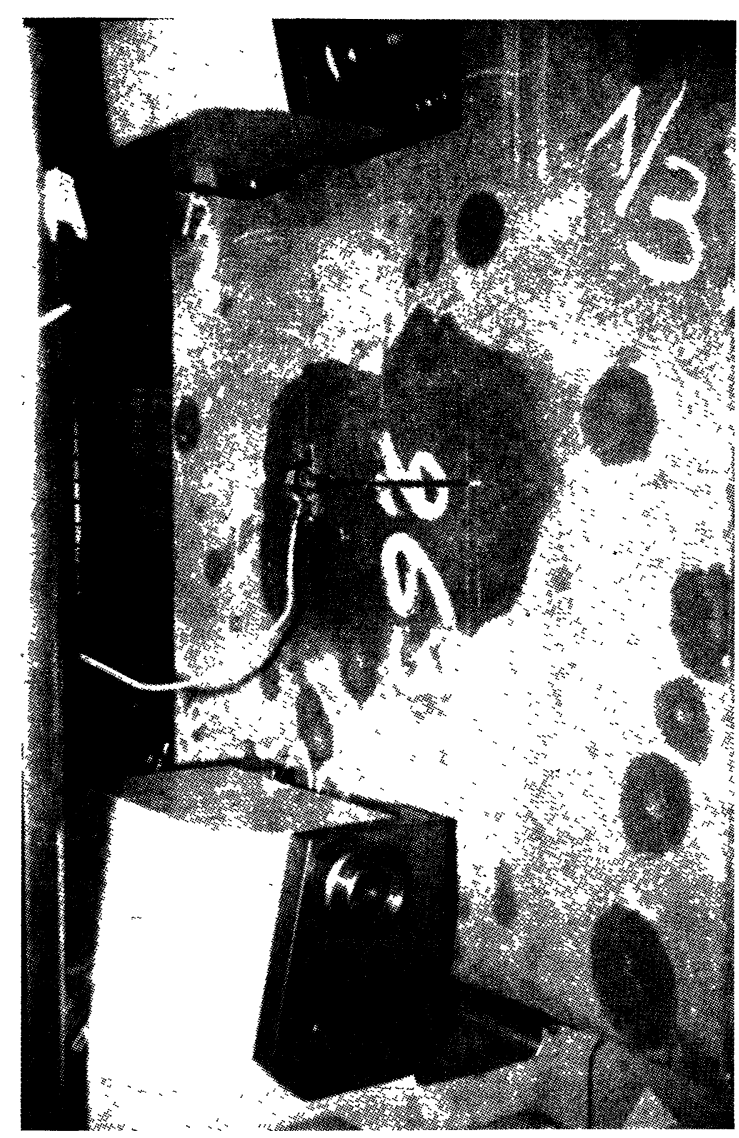

Рното 1.

La mesure de l'écartement du défaut se fait à $20 \mathrm{~mm}$ en arrière du fond de celui-ci (Fig. 5) car on a cherché à éviter les perturbations dans la zone plastifiée située en avant de ce défaut, perturbations apportées par les trous de fixation de cales du capteur. Remarquons que le déplacement de ce défaut est dans cette position, différent de celui de l'extrémité et il faut étalonner ou calculer la valeur de l'écartement de fissuration en fonction du déplacement $V$ des lèvres du capteur.

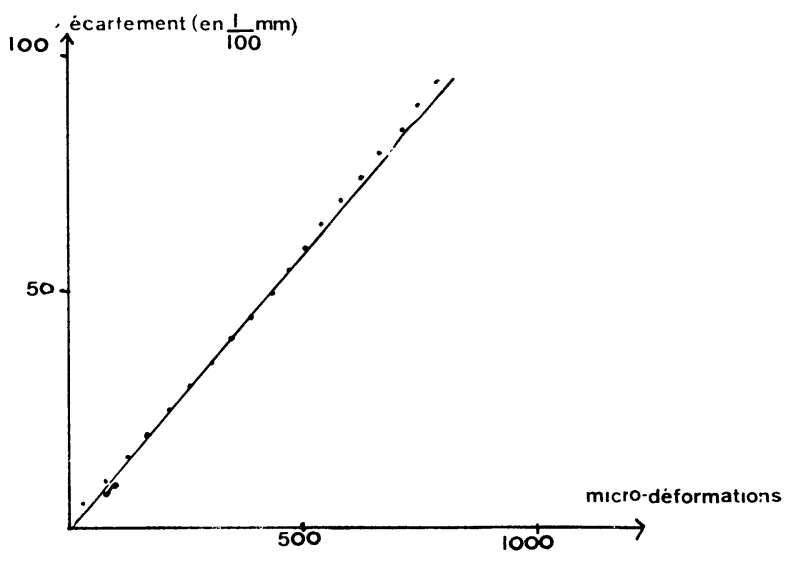

Fig. 6. - Courbe d'étalonnage du capteur.

Pour cela on suppose qu'il existe un centre de rotation fictif dans le ligament de la largeur $W-a$ (2 $W$ étant la largeur de la plaque et $2 a$ étant la longueur du défaut) qui existe sans le défaut. On admet que ce centre de rotation est situé à une distance $(W-a) / r ; r$ étant une constante appelée «facteur de rotation $"$.

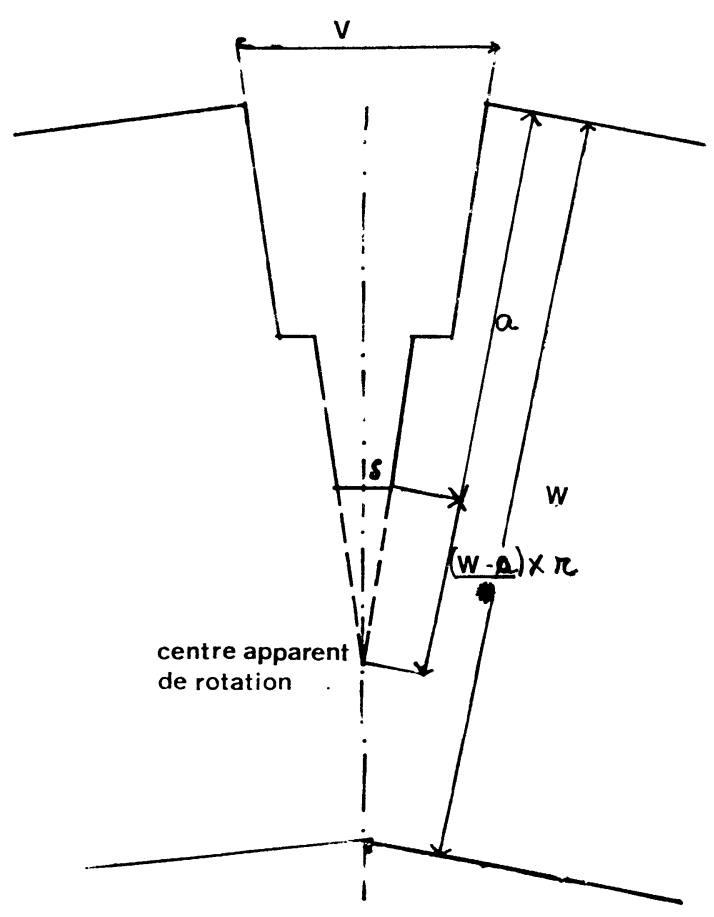

Fig. 7. - Relation géométrique entre l'écartement de fissure et les lèvres de l'entaille.

En considérant la figure géométrique 7, on obtient la relation qui lie l'écartement de fissure au déplacement des lèvres du capteur.

$$
\delta=\frac{V}{1+\frac{b \times r}{(W-a)}} .
$$


La valeur de la constante $r$ est donnée par des auteurs [10] comme étant égale à $1 / 3$, valeur que nous retiendrons.

$b$ est l'épaisseur des cales nécessaires à la fixation du capteur.

Le tableau I rassemble les caractéristiques géométriques des éprouvettes utilisées et la valeur du coefficient $M$ et $M^{\prime}$ permettant d'obtenir les valeurs de l'écartement de fissuration $\delta$ en fonction de l'écartement $V$ ou de la déformation $\varepsilon$ du capteur.

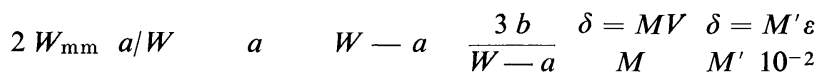

$\begin{array}{rrrrlll}\overline{-} & \overline{1} & \overline{-} & \overline{1} & \overline{-} & \overline{-} & \overline{0} \\ 300 & 1 / 5 & 30 & 120 & 0,52 & 0,67 & 0,76 \\ 300 & 1 / 4 & 37,5 & 112,5 & 0,58 & 0,66 & 0,75 \\ 300 & 1 / 3 & 50 & 100 & 0,6 & 0,62 & 0,71 \\ 300 & 1 / 2 & 75 & 75 & 0,78 & 0,56 & 0,70 \\ 400 & 1 / 3 & 67 & 133 & 0,45 & 0,69 & 0,78 \\ 500 & 1 / 3 & 83 & 162 & 0,37 & 0,735 & 0,83 \\ 600 & 1 / 3 & 100 & 200 & 0,30 & 0,77 & 0,88 \\ 600 & 1 / 2 & 150 & 150 & 0,40 & 0,715 & 0,80 \\ 600 & 2 / 3 & 200 & 100 & 0,6 & 0,62 & 0,70 \\ 800 & 3 / 4 & 300 & 100 & 0,60 & 0,62 & 0,70 \\ 800 & 7 / 8 & 350 & 50 & 1,2 & 0,45 & 0,51\end{array}$

On enregistre simultanément le diagramme des efforts appliqués en fonction d'une part du dépla-

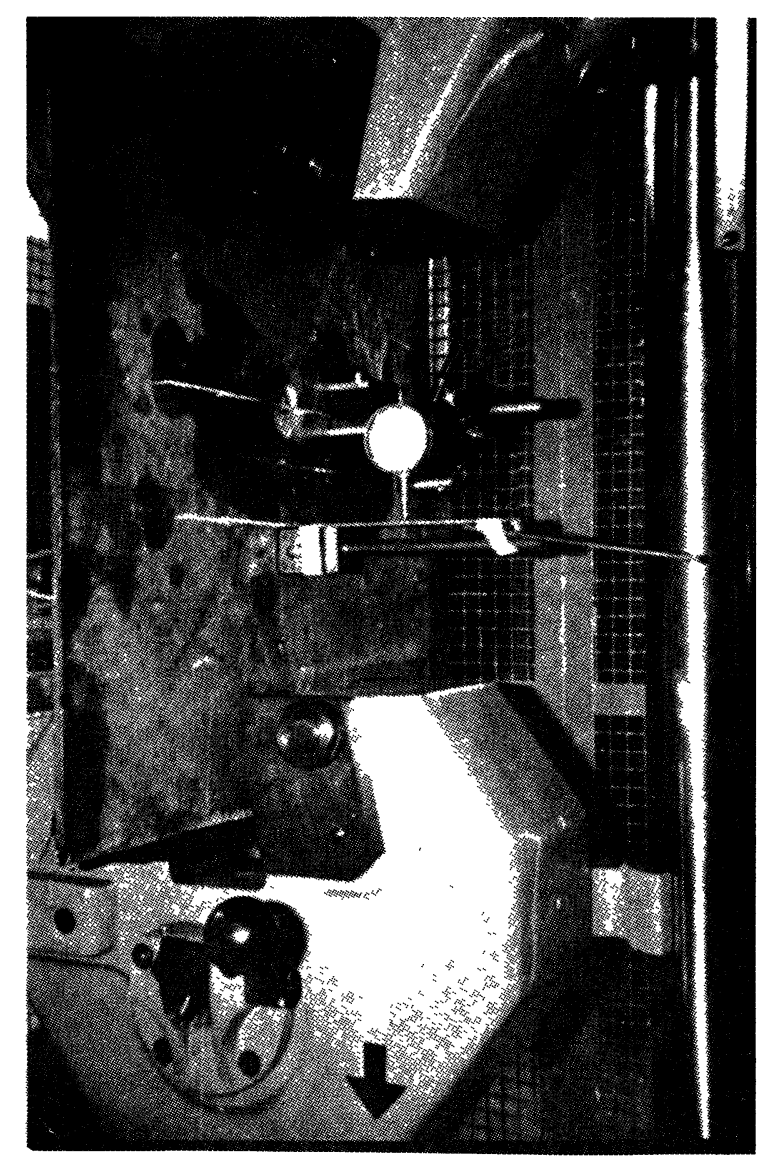

Рното 2. cement des branches du capteur et d'autre part de l'allongement global de part et d'autre du défaut sur une base de $100 \mathrm{~mm}$ à l'aide d'un comparateur au $1 / 100$ (photo $\mathrm{n}^{0} 2$ ). Les figures 8 et 9 donnent un exemple des courbes de l'effort appliqué en fonction de l'écartement ou de l'allongement global.

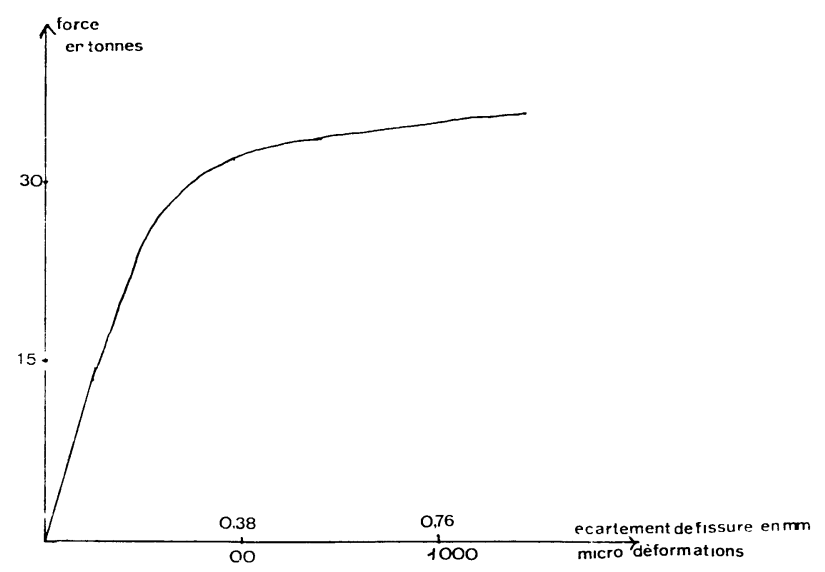

Fig. 8. - Courbe force-écartement de fissure plaque de $300 \mathrm{~mm} \mathrm{~A} / \mathrm{W} 1 / 6$.

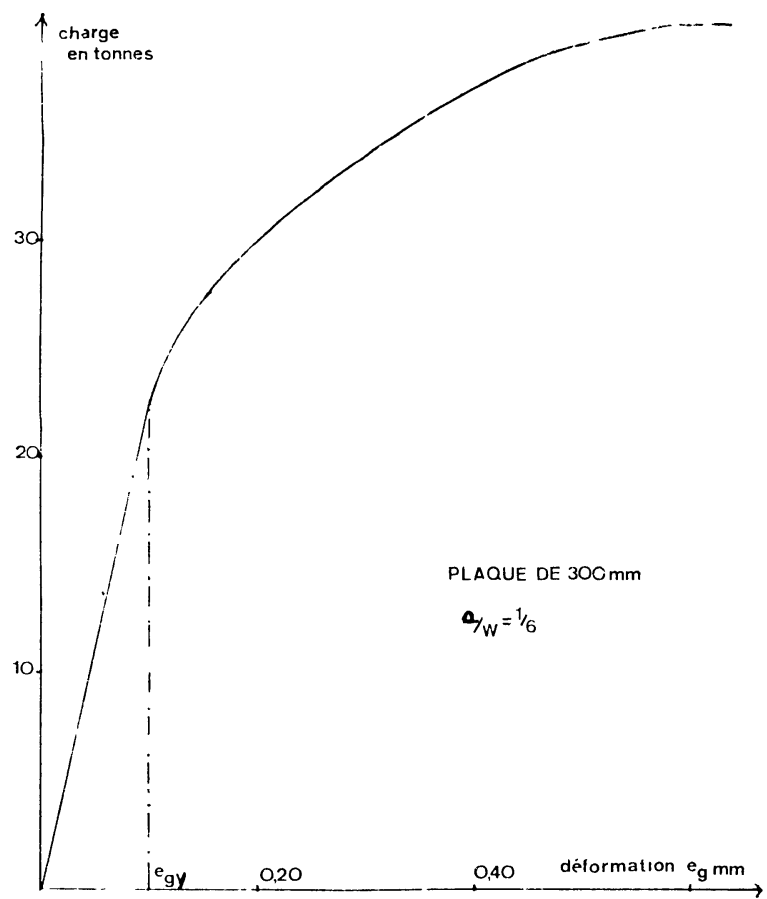

Fig. 9. - Courbe charge-déformation globale autour du défaut.

La courbe présente une similitude avec une courbe de traction conventionnelle. L'exemple est pris sur une plaque carrée de $300 \mathrm{~mm}$ et de rapport $a / W=1 / 6$.

On peut définir un allongement élastique $e_{\mathrm{g} y}$ sur la courbe $F=f\left(e_{\mathrm{g}}\right)$ à l'endroit où celle-ci perd sa linéarité.

2.3 Résultats expérimentaux. - Les éq. (4) et (8) nous indiquent que l'écartement non dimensionnel de fissuration $\Phi$ et la déformation globale 
autour du défaut $e_{\mathrm{gy}}$ sont liés par l'intermédiaire du rapport de la contrainte appliquée sur la limite élastique $\left(\sigma / \sigma_{y}\right)$. Les courbes $\Phi=\left(f\left(e_{\mathrm{g}} / e_{\mathrm{gy}}\right)\right)$ ont été calculées pour plusieurs valeurs du rapport longueur de fissure/longueur de jauge (Fig. 10). On remarque

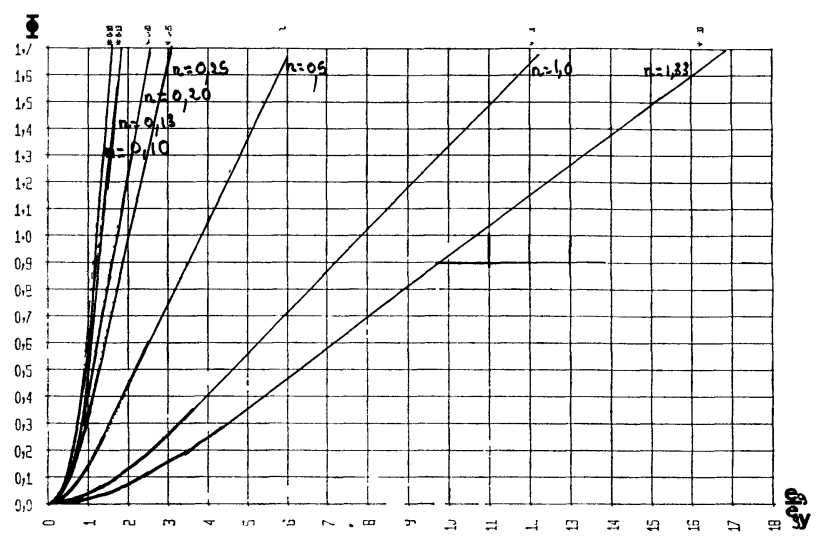

FIG. 10. - Courbes théoriques (éq. (4) et (8)).

que ces courbes deviennent rapidement linéaires et la pente de la courbe dépend de ce rapport longueur de fissure/longueur de jauge (c.-à.-d. la longueur de base de mesure de la déformation globale $-100 \mathrm{~mm}$ dans tous nos essais -). Nos résultats expérimentaux se proposent la vérification de cette linéarité entre l'écartement de fissuration et le degré d'avancement de la déformation caractérisée par le rapport $e_{\mathrm{g}} / e_{\mathrm{g} y}$.

a) Pour cela une première série d'essais a utilisé des éprouvettes de $300 \mathrm{~mm}$ de côté dans lesquelles nous avons fait varier le rapport longueur du défaut sur largeur de l'éprouvette. Ce rapport a pris successivement les valeurs $1 / 6,1 / 5,1 / 4,1 / 3,1 / 2$.

La figure 11 indique bien pour chaque valeur du rapport longueur du défaut/longueur de jauge une linéarité des courbes et que les 4 courbes pour les

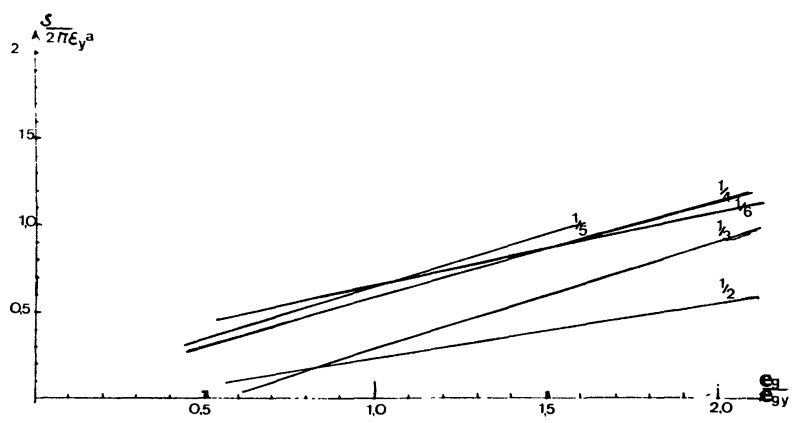

FIG. 11. - Relations expérimentales entre l'écartement de fissure et la déformation globale autour du défaut pour diverses valeurs de longueur de défaut. La dimension des plaques est constante et égale à $300 \mathrm{~mm}$.

rapports $1 / 3,1 / 4,1 / 5$ et $1 / 6$ sont groupées dans une même bande de dispersion indiquant une certaine indépendance vis-à-vis de ce rapport $a / W$. On remarque cependant que la pente de la courbe $1 / 2$ est légèrement plus faible que les autres, ce qui peut traduire l'influence du rapport longueur de défaut sur longueur de jauge.

Pour cette plaque $(a / w=1 / 2)$ notons d'une part que la valeur relevée de la déformation globale élastique est deux fois plus élevée que pour les autres plaques, d'autre part que le «ligament » existant entre le bord et le défaut est le plus petit et que la pente de la courbe est voisine de celles des courbes relatives aux plaques possédant de plus faibles « ligaments » sans défaut, ce qui laisse supposer que l'influence de l'envahissement des bords de l'éprouvette par la zone plastique créée sous le défaut n'est alors plus négligeable.

Pour connaître cette influence, nous avons cherché à préciser expérimentalement la forme de cette zone. Par examen en lumière rasante on voit nettement cette zone plastique: on remarque que deux zones de déformation s'étendent dans une direction voisine de $45^{\circ}$ des deux axes et qu'un accroissement de déformation augmente la largeur de ces zones plastiques dans la direction $x$.

La plaque ayant des dimensions finies, la zone plastique finit par atteindre les bords de l'éprouvette, on peut constater alors que l'écartement de fissure devient égal à la déformation globale, ce qui oblige nécessairement à limiter les investigations en dessous de la déformation plastique généralisée en effet lorsque $a_{1}=W$ l'éq. (4 bis) entraîne $\sigma / \sigma_{y}>1$ ce qui oblige à tenir compte de l'écrouissage).

Un essai utilisant 9 jauges de contraintes réparties dans la zone plastique comme l'indique la figure 12 a permis de préciser dans une plaque de $200 \mathrm{~mm}$ de côté, l'évolution des déformations au cours d'un essai.

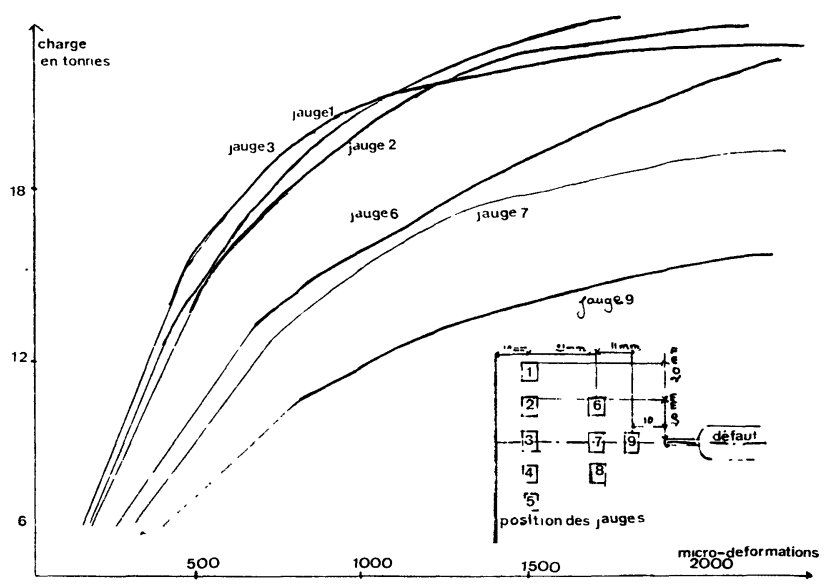

EVOLUTION DES DEFORMATIONS DANS LA ZONE PLASTIOUE AU COURS DU CHARGEMENT

FIg. 12. - Evolution des déformations dans la zone plastique au cours du chargement.

Les courbes charge-déformation relatives aux jauges 1 , 2 et 3 , et 6,7 et 8 sont assez proches et indiquent un avancement parallèle et symétrique des bords de la zone plastique et une distribution non homogène des déformations. La répartition en fond du défaut 
est précisée à l'aide de chaînette constituée de 5 petites jauges parallèles à la direction de sollicitation (Fig. 13). Une anomalie sur la courbe indique l'envahissement de la jauge par la zone plastique, cet effet est masqué par l'éloignement dans les autres jauges.

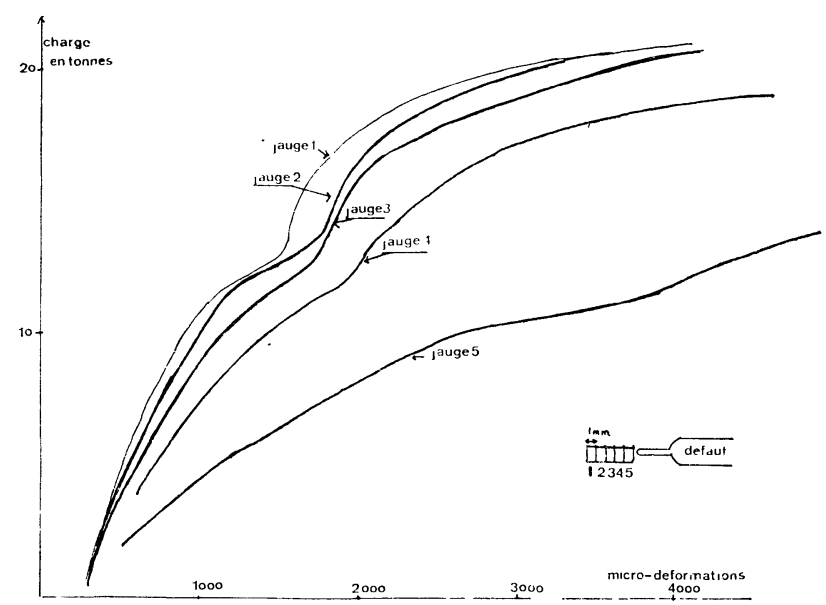

FIg. 13. - Mesure des déformations à proximité du fond d'entaille.

b) Dans un deuxième groupe d'essais, nous avons maintenu le rapport longueur de défaut sur largeur d'éprouvette constant et égal à $1 / 3$. Nous avons fait varier les dimensions géométriques des éprouvettes en utilisant des plaques carrées de $300 \mathrm{~mm}$ de côté, $400 \mathrm{~mm}, 500 \mathrm{~mm}$ et $600 \mathrm{~mm}$.

De la même façon on obtient des relations linéaires entre l'écartement de fissure non dimensionnel et le rapport $e_{\mathrm{g}} / e_{\mathrm{g} y}$, les droites sont cependant situées dans une bande de dispersion un peu moins large et on note peu l'influence du rapport longueur du défaut/longueur de jauge (Fig. 14).

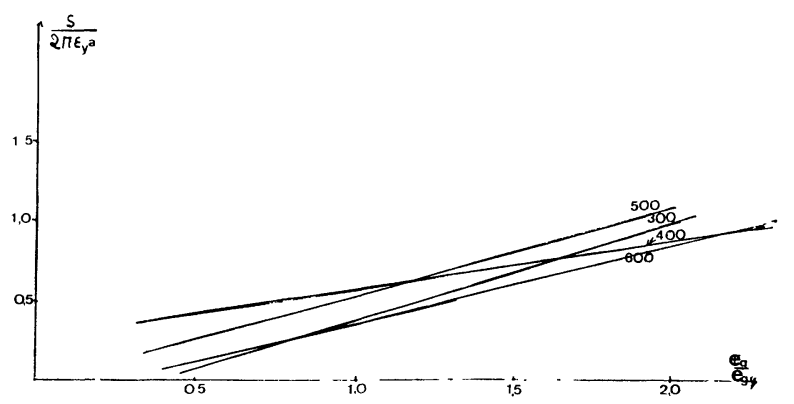

Fig. 14. - Influence de la dimension des plaques sur les courbes d'écartement de fissure en fonction de la déformation. Le rapport longueur de défaut sur largeur est constant et égal à $1 / 3$.

c) Il faut noter une diminution importante de la pente de la droite lorsque les défauts deviennent très grands (Fig. 16), et les essais conduisent d'autre part à un phénomène de gauchissement des plaques.

Cette diminution importante de la pente de la courbe est due à une valeur élevée du rapport longueur du

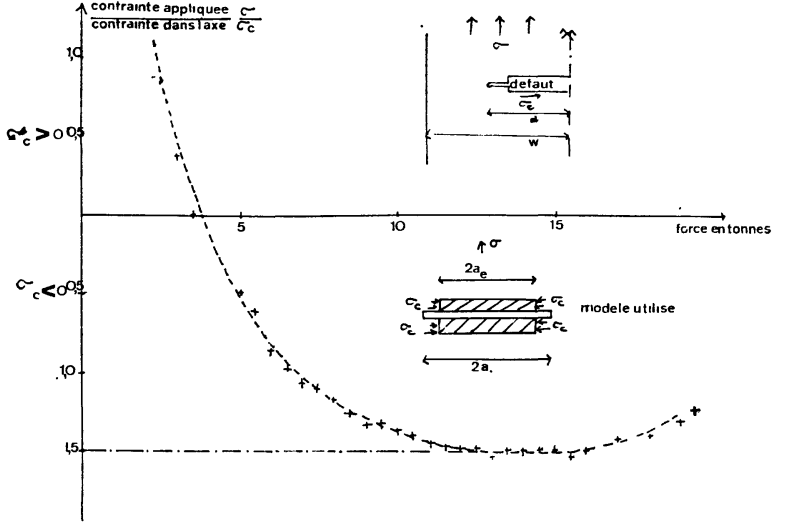

Fig. 15. - Variation de la constante dans l'axe du défaut.

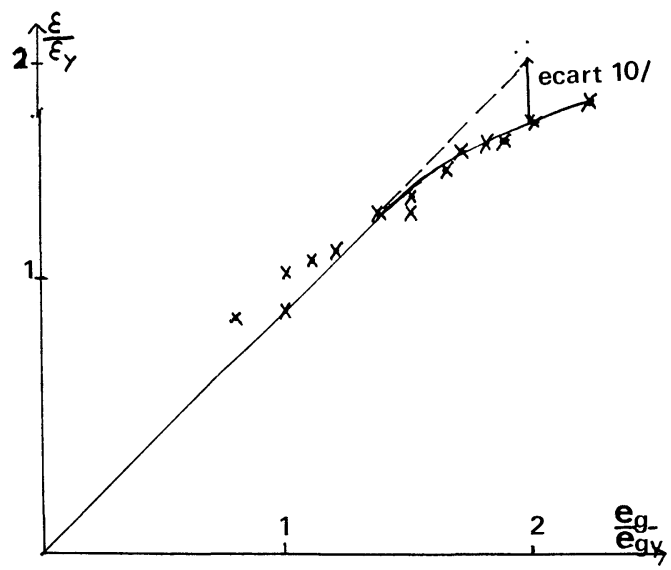

Fig. 16. - Courbes d'écartement de fissure-déformation relative aux grands défauts.

défaut sur longueur de jauge, diminution mise en évidence sur les courbes théoriques et l'influence importante d'effet de bord; il faut remarquer que le risque de rupture par ouverture des lèvres du défaut diminue fortement mais on augmente alors les risques de rupture " par glissement vis » des lèvres du défaut (rupture du mode III).

On observe une bonne concordance entre les résultats théoriques de la figure 10 et nos résultats expérimentaux ainsi que la même dépendance vis-à-vis de la longueur de jauge.

Ce phénomène de gauchissement a été étudié par J. R. Dixon et J. S. Strannigan [11] et est dû à l'existence d'une contrainte $\sigma_{\mathrm{c}}$ de direction parallèle à l'axe du défaut, contrainte de compression le plus souvent.

En effectuant le rapprochement du phénomène observé avec le flambement d'une poutre articulée à ses deux extrémités et soumise à un effort de compression d'après la théorie d'Euler on obtient :

$$
\sigma_{\mathrm{c}}=\frac{\pi^{2} E}{\gamma^{2}}
$$


$\gamma$ étant l'élancement de la poutre. Dans le cas présent, le rayon de giration $r$ d'une poutre de section rectangulaire de largeur $b$, d'épaisseur $t$ étant donné par

$$
r^{2}=\frac{t^{2}}{12}
$$

nous obtenons nécessairement

$$
\gamma^{2}=\frac{(2 a)^{2}}{t^{2} / 21}=\frac{48 a^{2}}{t^{2}}
$$

et

$$
\sigma_{\mathrm{c}}=\frac{\pi^{2} E t^{2}}{48 a^{2}}
$$

En prenant $\left(\sigma_{\mathrm{c}}\right)=1,5 \sigma$ (valeur que nous avons relevée dans un essai sur plaque comportant des jauges parallèles à la direction du défaut) (Fig. 15) $\sigma$ étant la contrainte nominale soit $F / 2 t W$.

On peut donc estimer la valeur de la contrainte critique

$$
\sigma_{\text {critique }}=1,5 \frac{\pi^{2}}{48 a_{\mathrm{e}}} \frac{E t^{2}}{2}
$$

Remplacer la longueur $2 a$ longueur réelle du défaut par $2 a_{\mathrm{e}}$ revient en fait à tenir compte du fait que la longueur libre de flambement n'est pas celle du défaut.

Dixon et Strannigan ont raisonnablement montré que l'on avait $a_{\mathrm{e}}$ compris entre $0,4 a$ et $0,6 a$.

$a_{\mathrm{e}}$ est la longueur de la poutre équivalente de notre figure 16. Ce phénomène est important dans les constructions, car bien que de grands défauts ne soient pas évidemment admissibles, il faut noter qu'en mécanique de la rupture une petite fissure prenant naissance sur le bord d'un orifice est assimilable à un défaut du diamètre de l'orifice, de même la propagation d'un défaut peut l'amener à une taille suffisante pour provoquer ce phénomène qui se caractérise par une déformation "en tonneau » de la construction.

Ainsi du point de vue de la mécanique de la rupture un défaut peut-il être en dessous de sa taille critique, mais toutefois lorsque l'épaisseur est faible posséder une longueur telle qu'elle provoque ce type de rupture après déformation.

3. Utilisation de la théorie de l'écartement critique de fissuration à la prévision de la taille des défauts critiques. - L'intérêt de la mécanique des ruptures est non seulement qu'elle procure une classification des matériaux par une valeur calculée de la ténacité (cette classification se fait habituellement par l'approche «température de transition »), mais qu'elle permet en outre théoriquement d'estimer la taille des défauts admissibles dans les constructions. Le développement rapide de nombreuses réalisations tant au point de vue de leurs tailles que de leur diversité a entraîné les bureaux d'études et les ingénieurs chargés de sélectionner les matériaux utilisés à prendre des précautions pour se prémunir du risque de rupture fragile.

Ces précautions de plus en plus draconniennes entraînent le rejet de matériaux de bonne qualité et le rebut de constructions possédant des défauts inférieurs à ce que l'on pourrait théoriquement admettre pour satisfaire aux normes dont la détermination est parfois empirique. Cela a une incidence sur le coût des constructions et ne permet pas de tirer parti au maximum des matériaux utilisés. Ces défauts rencontrés dans les matériaux peuvent être dus aux procédés de fabrication (laminage par exemple), aux procédés de constructions (soudage), ils peuvent être très fins (fissure) ou volumineux (nids d'inclusions, soufflures). Il faut cependant remarquer que la mécanique des ruptures ne se soucie pas de la spécifité du défaut : en effet, on considère comme ayant affaire un défaut plan, de forme géométrique simple (généralement elliptique) avec une acuité très grande. Ce défaut est situé d'autre part dans un matériau aux propriétés élasto-plastiques homogènes et isotropes. Signalons une étude de P. C. Paris et G. C. Sih [12] en mécanique linéaire des ruptures sur quelques formes géométriques de défauts.

Rappelons le résultat de la mécanique des ruptures sur la relation reliant la taille de défaut critique à l'énergie de propagation de fissure $G_{c}$ dans les conditions de contrainte plane.

$$
a_{\mathrm{c}}=\frac{E G_{\mathrm{c}}}{\pi \sigma_{y}^{2}}
$$

en tenant compte de la relation (7) $G_{c}=\sigma_{y} \delta$, on aboutit à une relation de la forme

$$
a_{\mathrm{c}}=\text { Cte } \times \frac{\delta_{\mathrm{c}}}{\mathrm{e}_{\mathrm{y}}} .
$$

La valeur de $\varepsilon$ et $\varepsilon_{y}$ peut être obtenue expérimentalement par la mesure de l'écartement de fissuration sur une longueur de jauge équivalente à la longueur de la micro-éprouvette de la théorie de Cottrell [15] [c.-à-d. 2 fois la valeur de l'écartement de fissure]

$$
\varepsilon=\frac{\partial \delta}{2 \delta}=\frac{1}{2} \log \delta
$$

Ce sont Wells [13] et Burdekin [14] qui ont signalé l'importance de ce facteur $\delta_{c} / \varepsilon_{y}$ dans la prévision de la taille des défauts pour les ruptures au-delà de la limite d'élasticité comme le facteur $\left(K_{1} \mathrm{c} / \sigma_{y}\right)^{2}$ l'est dans les aciers à haute limite d'élasticité.

La relation (13) peut s'écrire sous la forme

$$
a_{\mathrm{c}}=\left[\frac{1}{2 \pi \Phi}\right]\left[\frac{\delta_{\mathrm{c}}}{\varepsilon_{y}}\right] .
$$

Donc à un niveau de déformation globale fixé par une valeur du rapport $e_{\mathrm{g}} / e_{\mathrm{g} y}$ la valeur de $\Phi$ obtenue sur les courbes $\Phi=f\left(e_{\mathrm{g}} / e_{\mathrm{g} y}\right)$ permet de calculer la constante de la relation (13). 
En utilisation de récipients sous pression, deux valeurs de pression sont importantes : la pression maximale de service qui va correspondre à une valeur du rapport $\sigma / \sigma_{y}$ égal à 0,64 et la pression atteinte lors de l'essai de timbrage qui va correspondre à une mise en pression une fois et demie plus grande que la pression de service, soit un rapport $\sigma / \sigma_{y} \simeq 0,95$.

Tant que $e_{\mathrm{g}} / e_{\mathrm{g} y}$ est inférieur à 2 , pratiquement les courbes $\left(\sigma / \sigma_{y}\right)$ et $\left(e_{\mathrm{g}} / e_{\mathrm{g}}\right)$ sont homothétiques, on peut donc remplacer les valeurs de $\sigma / \sigma_{y}$ par celle de $\left(e_{\mathrm{g}} / e_{\mathrm{gy}}\right)$ c'est ce qui fait l'intérêt de l'utilisation de la déformation globale autour du défaut car pratiquement dans le même domaine $\varepsilon / \varepsilon_{y}$ s'identifie à $e_{\mathrm{g}} / e_{\mathrm{g} y}$ (Fig. 17).

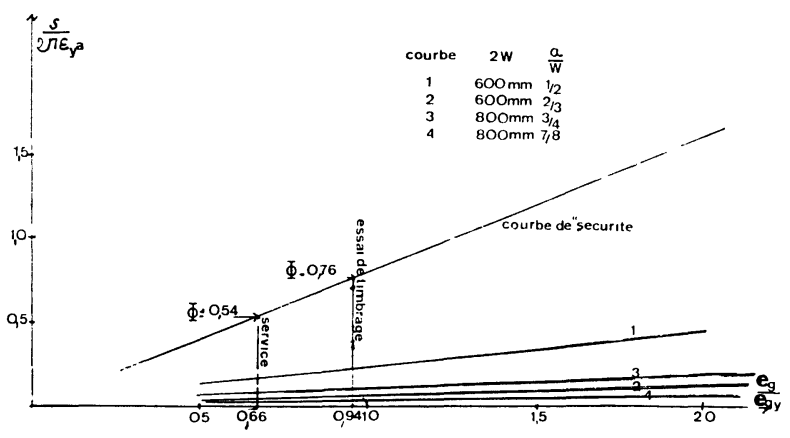

COURBES DECARTEMENTDE FISSURE-ceformation RELATIVE AUX GRANDS DEFaUtS

FIG. 17. - Relation expérimentale entre $e_{\mathrm{g}} / e_{\mathrm{g} y}$ et $\varepsilon / \varepsilon_{\mathrm{g}}$.

Nous avons choisi une courbe "sécurité " qui correspond à la courbe supérieure (Fig. 16) de la bande de dispersion des essais que nous avons réalisé. Cette courbe de "sécurité » nous donne pour les valeurs de $e_{\mathrm{g}} / e_{\mathrm{g} y}=0,64$ une valeur de $\Phi_{1}=0,59$ ce qui entraîne $a_{\mathrm{c}_{1}}=0,285 \times\left(\delta_{\mathrm{c}} / \varepsilon_{y}\right)$ de la même manière pour une valeur de $e_{\mathrm{g}} / e_{\mathrm{g} y} \simeq 0,95, \Phi_{2}=0,77$ et $a_{\mathrm{c}_{2}}=0,213\left(\delta_{\mathrm{c}} / \varepsilon_{\mathrm{y}}\right)$ (Fig. 16). Dans les conditions de l'analyse précédente $\left(e_{\mathrm{g}} / e_{\mathrm{g} y}<2\right)$, les valeurs de $S_{\mathrm{c}}$ sont au maximum de $0,160 \mathrm{~mm}$ et les valeurs de $a_{\mathrm{c}}$ sont respectivement $a_{\mathrm{c}_{1}}=44 \mathrm{~mm}$ et $a_{\mathrm{c}_{2}}=52 \mathrm{~mm}$.

Ces conditions sont plus sévères que celles de Burdekin et Daves [14] qui préconisent respectivement les coefficients 0,5 et 0,25 . On peut d'ailleurs augmenter la sévérité des conditions en introduisant les facteurs de concentration de contrainte qui sont introduits inévitablement notamment au piètement des récipients soudés.

4. Conclusions. - Nous avons tenté de relier l'écartement critique de fissuration au défaut admissible dans les constructions métalliques en acier à basse et moyenne résistance. Une courbe de "sécurité " nous indique les coefficients à utiliser lors de l'essai de timbrage et de la mise en service de l'appareil. Ce résultat pour intéressant qu'il soit nous paraît cependant peu complet et il nous semble intéressant de poursuivre en étudiant les effets de l'acuité et de la spécificité des défauts, de la triaxialité, des effets des caractéristiques d'écrouissage du matériau, de la température d'essai. Il ne faut pas de plus perdre de vue que l'établissement de telles courbes ne peut se faire qu'après confrontation des résultats de divers laboratoires pour être utilisables.

\section{Bibliographie}

[1] Wells, A. A., Welding Research 7 (1953) 34

[2] Wessel, E. T., Clark, W. G., Pryle Fracture mechanics technology, Applied to heavy section steel fracture 2nd international conference on fracture Brighton, 13-18 avril (1972) p. 72.

[3] Tiffany, C. F. et Masters, S. N., Applied fracture mechanics, Fracture Toughness Testing and its applications, p. 249-272 ASTM STP 381.

[4] Wells, A. A., Application of fracture mechanics at and beyond general yielding. British Welding Journal 10-11 (1963), 563-570.

[5] Burdekin, F. M. et Stone, D. E. W., « The crack opening displacement approach to fracture mechanics Yielding materials », Journal of strain Analysis 1-2 (1966) 145 à 153.

[6] Dugdale, D. S., Yielding of steel sheets containing slits, J. Mech. Phys. solids 18 (1960) 100.

[7] BARENBLATT, The mathematical theory of equilibrium cracks in brittle fracture. Adv. appl. Mechanics, 7 (1962) (55).

[8] Pluvinage (G.), Aspects théoriques et expérimentaux de l'ouverture critique de fissure. Journée de l'ATS, Dunkerque, mai (1971).
[9] Randall, P. N. et Merkle, J. G., " Cross strain crack tolerance of steel », Nuclear engineening and design 17 (1971) 46-63.

[10] Ingham, T., Egan, G. R., Elliot, D., Harrisson, T. C., The effect of Geometry on the interpretation of COD Test Data, C 54-71 Inst. Mech. Engnrs p. 200-208 (1971).

[11] Dixon, J. R. et Stranningam, J. S., « Stress distribution and buckling in thin sheets with central slits ». Fracture Brighton 1969 p. 105-118.

[12] Paris, P. C. et Sir, G. S., « Stress analysis of cracks», ASTM STP 381, p. 30-83.

[13] Wells, A. A., The specification of permissible defect sizes in welded metal p. 868-880. Fracture 1969. Proceeding of the Second International Conference on the fracture Brighton, avril (1969).

[14] Burdekin, F. M., DAwes, M. G., Practical use of linear elastic and Yielding fracture mechanics with particular reference to pressure Vessels, p. 28-37 Inst. Mech. Engnrs (1971).

[15] Cottrell, A. M., Proc. R. Soc. 285 (1965) 10. 